\title{
MARINE POLLUTION IN EUROPEAN COASTAL WATERS MONITORED BY THE ERS-2 SAR: A COMPREHENSIVE STATISTICAL ANALYSIS
}

\author{
Martin Gade* and José M. Redondo** \\ * Universität Hamburg, Institut für Meereskunde, Troplowitzstr. 7, 22529 Hamburg, Germany \\ Phone: +49(0)40 428385450 Fax: +49(0)40 428385713 E-mail: gade@ifm.uni-hamburg.de \\ ** Dipartament de Fisica Applicada, Universitat Politècnica de Catalunya, 08034 Barcelona, Spain \\ Phone: +343 4017984 Fax: +343 4016090 E-mail: redondo@etseccpb.upc.es
}

\begin{abstract}
The oil pollution of the southern Baltic Sea, the North Sea, and the northwestern Mediterranean Sea has been studied within a two-year period from December 1996 until November 1998. In total, we have analyzed more than 700 synthetic aperture radar (SAR) images, which have been acquired over the three test areas by the Second European Remote Sensing Satellite (ERS-2). In this paper, we present the results of our statistical analyses. Moreover, we introduce advanced image processing techniques for the classification of the observed radar signatures, namely the calculation of the fractal dimension, and discuss the possibility of identifying different types of oceanic phenomena.
\end{abstract}

\section{INTRODUCTION}

Within the project "Clean Seas", which is funded by the European Commission, three test areas in European marginal waters, namely the southern Baltic Sea and North Sea and the northwestern Mediterranean (for details see [1]), were chosen for the remote sensing of marine pollution. The test areas include some main ship routes and the estuaries of the major rivers Oder (Baltic Sea), Thames, Rhine (North Sea), and Rhone (Mediterranean). In total a sea surface area of more than 250,000 $\mathrm{km}^{2}$ has been monitored. From each of the three test areas 15 SAR frames [1] have routinely been acquired by the synthetic aperture radar (SAR) aboard the Second European Remote Sensing Satellite (ERS-2), so that a total of 709 SAR images could be used for our analyses. Since oil spills dampen the small-scale surface waves, which are responsible for the radar backscattering from the water surface, they are visible as dark patches in SAR imagery.

However, some oceanic and atmospheric phenomena may cause signatures similar to those of oil spills, so that any automated oil detection algorithm using radar imagery may cope with false alarms. For that reason, we analyzed the SAR images by eye, in order to ensure maximum confidence of the statistics to be produced. The SAR images were processed at a resolution of $100 \mathrm{~m}$ and were provided by the RApid Information Dissemination System (RAIDS) SAR processing facility in West Freugh, UK.

\section{STATISTICAL RESULTS}

During the two years of our studies, 220 SAR images have been acquired over the Baltic Sea test area, 207 SAR images over the North Sea test area, and 282 SAR images over the test area in the northwestern Mediterranean Sea. We have analyzed every SAR image with respect to the occurrence of marine oil pollution. The locations and sizes of the detected oil pollution have been calculated and catalogued. As a result, we included circles into the three maps shown in Fig. 1 at the locations of the detected oil spills. The sizes of the circles are proportional to those of the oil spills, ranging from $0.1 \mathrm{~km}^{2}$ to $56 \mathrm{~km}^{2}$.

The main pollution occurs along the main ship traffic routes, which is most obvious in the Baltic Sea (see the left panel in Fig. 1) going from southwest to northeast. This effect is less pronounced in the North Sea test area, because of the overall dense ship traffic. In the northwestern Mediterranean, however, main ship traffic routes may also be inferred from the locations of detected oil pollution. However, the highest occurrence of marine oil pollution was found in the northwestern Mediterranean, south of Barcelona, where the outflow of the river Llobregat seems to cause a high incidence of pollution in that particular area. In total, we detected 675 oil spills from which 122 covered a sea area of more than $5 \mathrm{~km}^{2}$ [1]. We found more oil spills during summer time (bright, i.e. green, yellow, and orange, circles) than during winter time (dark, i.e. red, blue, and purple, circles).

In order to better demonstrate the spatial distribution of the detected marine oil pollution in the northwestern Mediterranean Sea we have calculated maps showing 

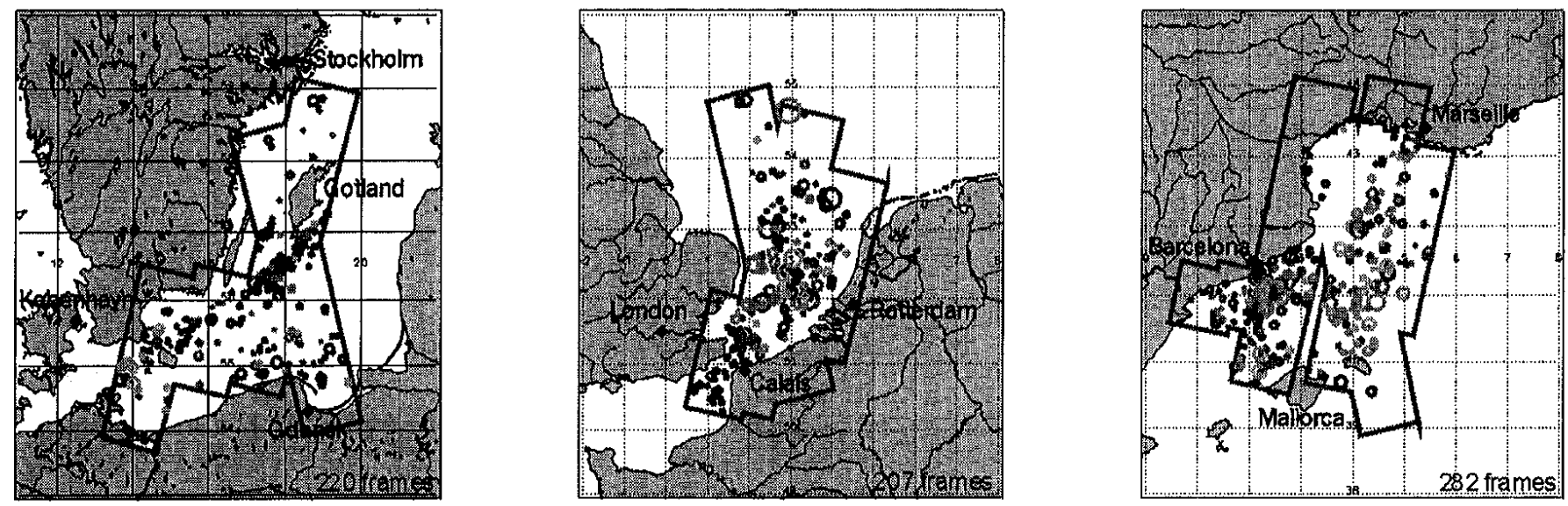

Logond: $=1 \mathrm{~km}^{2} \mathrm{O}=10 \mathrm{~km}^{2} \mathrm{O}=25 \mathrm{~km}^{2} \mathrm{O}=50 \mathrm{~km}^{2}$

JF MAM J J A S OND

Fig. 1: Maps of the three test areas Baltic Sea (left), North Sea (middle), and Mediterranean (right). The circles denote the locations of the detected oil spills, the circle sizes are proportional to the respective sizes of the oil spills, and the colorcoding gives the time of the year (namely the month) when the pollution has been detected. Note that the main pollution occurs along the main ship traffic routes and that we detected more oil spills during summer time (bright, i.e. green, yellow, and orange, circles) than during winter time (dark, i.e. red, blue, and purple, circles).

contour plots of the mean spill-covered area per SAR image. As an example, our results for the northwetsern Mediterranean Sea are depicted in Fig. 2. For the upper panel only oil pollution detected during winter time (October through March) has been taken into account, and for the lower panel only pollution detected during summer time (April through September). Each plot has been generated by determining the local (normalized) percentage of the total spill-covered area (i.e., the sum of the sizes of all detected oil spills divided by the area of the grid cell, which was chosen to be $20^{\prime}$ by $20^{\prime}$ ). As a maximum, we found mean polluted sea surface areas of more than $4000 \mathrm{~m}^{2}$ per $\mathrm{km}^{2}$ off the mouth of the river Llobregat near Barcelona.

It is noteworthy that the measured pollution is higher during summer time is higher than during winter time, which we attribute to the fact that the visibility of any marine pollution is higher in summer because of the overall lower (mean) wind speed and wave height. Thus, any oil pollution may be easier to detect because of the wind speed dependence of the visibility of oil spills on SAR images [1,2]. Especially during summer time (lower panel), the areas of highest pollution can easily be delineated: (1) south of Barcelona, where the outflow of the river Llobregat is driven southwards by the local currents, (2) along the main ship traffic routes going from Barcelona southwards and eastwards and from Marseille towards southwest, (3) around the harbor of Marseille, and (4) south of Tarragona, between Barcelona and the mouth of the river Ebro.

\section{CALCULATION OF FRACTAL DIMENSIONS}

Some of the theory relating fractal analysis to the turbulence is presented in [3]. Recently, fractal (image) analysis has become a rapidly evolving research field with various approaches, ranging from mathematical to experimental. In this paper we present a basic method for identifying different dynamical processes that might influence the radar backscattering from the ocean surface to demonstrate the capability of fractal analysis of improving existing oil pollution detection algorithms.

We used a box-counting algorithm to detect the selfsimilar characteristics for different SAR-image intensity levels. This purely geometrical description may be related to dynamical (oceanic and atmospheric) processes assuming that an energy transfer generated at a certain range of scales will affect the ocean surface at the same scales. The best geometrical characterization of a multifractal set showing different fractal dimensions $D$ for different SAR-image intensities $i$ is given by the maximum fractal dimension; however, relevant information may also be obtained from the complete function $D(i)$.

The fractal dimension $D(I)$ as a function of intensity $I$ may be calculated using

$$
D(i)=-\frac{\log N(i)}{\log e}
$$

where $N(i)$ is the number of boxes of size $e$ needed to cover the contour at a SAR intensity (i.e. radar backscattering) level $i$. The algorithm operates by dividing the 
digitized (two-dimensional) surface into smaller and smaller square boxes and by counting the number of them which have values close to the respective SAR intensity $i$.

Let us assume a convoluted line, which is embedded in a plane (that is why it is usually referred to as $D_{2}$, or
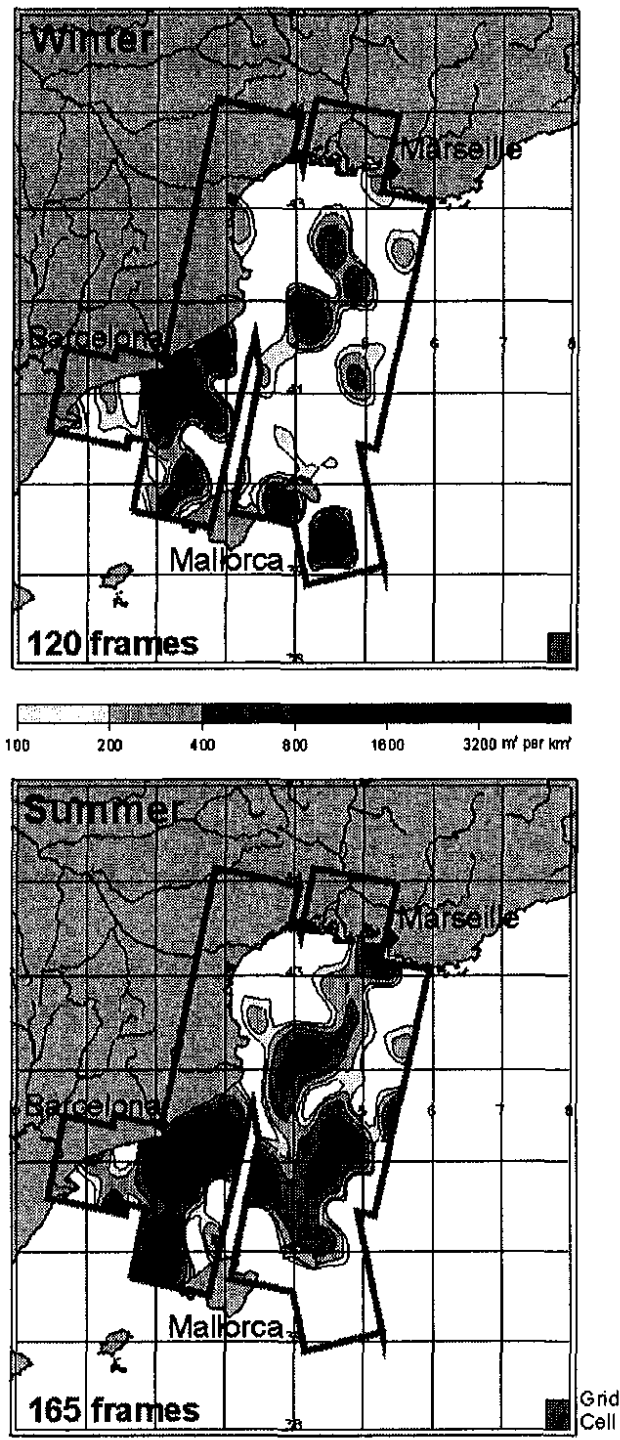

Fig.2: Contour plots denoting the mean spill-covered area in the northwestern Mediterranean Sea as retrieved from SAR images acquired between December ' 96 and November ' 98 . The mean (detected) pollution is higher during summer time (lower panel: April through September) than during winter time (upper panel: October through March). For each grid cell (20' by $20^{\prime}$ ) the total slick-covered area has been weighted with the number of acquired SAR frames. fractal dimension within an Euclidean plane of dimension 2). If it is a single Euclidean line, its (non fractal) dimension will be one. If it fills the entire plane its dimension will be two. The box-counting algorithm divides the embedding Euclidean plane in smaller and smaller boxes (by dividing the initial length $L_{0}$ of the SAR image by $n$, which is the recurrence level of the iteration). For each box of size $L_{0} / n$ it is then decided if the convoluted line, which is analyzed, is intersecting that box. The number $N(I)$ is the number of boxes which were intersected by the convoluted line (at intensity level $i$ ). Finally, we plot $N$ versus $L_{0} / n$ (i.e., the size $e$ of the box) in a log-log plot, and the slope of that curve, within reasonable experimental limits, gives the fractal dimension. Note that the sign of the fractal dimension is not relevant. As a demonstration of the calculation of fractal dimensions Fig. 3 shows a loglog plot of the number of boxes $N(i)$ covering the convoluted line at a certain intensity level $i$ as a function of the box size $e$ (in pixels).

The calculation of $N(i)$ has to be done for different contour levels corresponding to different SAR intensity levels $i$. However, for practical purposes it is enough to check at the edges of the boxes whether there is any pixel with the desired intensity level, except in very fragmented convoluted lines.

We show in Fig. 4 five different sections of ERS-2 SAR images corresponding to different oceanic and atmospheric phenomena. Also plotted are the respective functions $D(i)$ without (dark, purple) and after applying a speckle-noise (Kuan) filter (bright, pink): a,b) anthropogenic oil spills of different shapes, $c$ ) atmospheric convective cells, d) biogenic sea slicks, and e) rain cells.

All curves shown in Fig. 4 exhibit a strong increase of

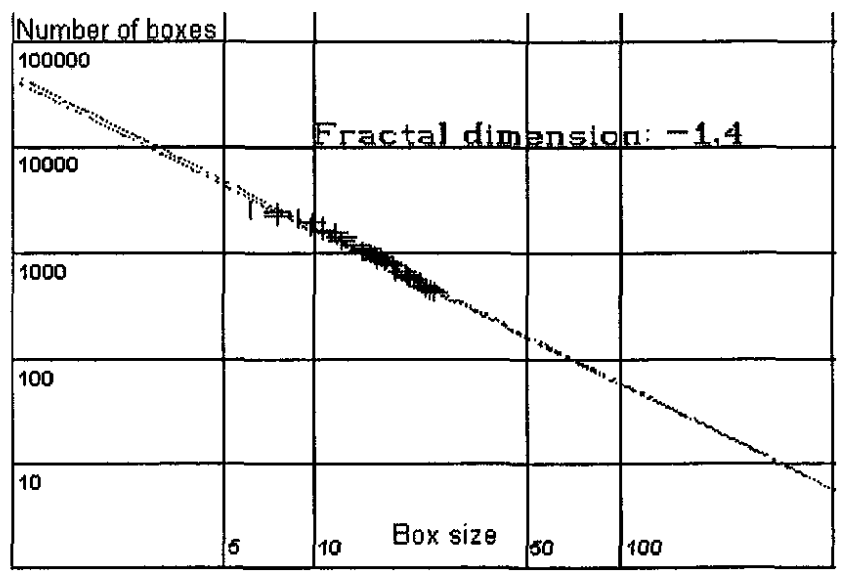

Fig. 3: Example for the calculation of the fractal dimension. In this log-log plot the number of bixes covering the convoluted line is plotted as a function of the box size (in pixels). 
$D(i)$ at low SAR image intensities (or normalized radar cross sections, NRCS) between $-30 \mathrm{~dB}$ and $-20 \mathrm{~dB}$ and a strong decrease at larger backscatter values between $-18 \mathrm{~dB}$ and $-5 \mathrm{~dB}$. Especially Panels $a), b$ ), and e) show a pronounced maximum at large NRCS values (i.e. at the right-handed side of the curve), which is due to the speckle noise, a typical characteristic of SAR images. However, the anthropogenic oil spills (Panels a) and b)) tend to exhibit a second peak at lower backscatter values. This second peak, which is best pronounced for the elongated spill shown in Panel a), has a smaller (maximum) fractal dimension. On the other hand, the windinduced patterns and natural slicks (Panels $c$ ) and d), respectively) show a broad (and larger) maximum of $D(i)$. The irregular-shaped rain cells (Panel $e)$ ), again, seem to cause a second peak at lower backscatter values. The "tail" at the high-backscatter ends of the curves in Panels b) and c) are due to ships, which are visible as bright spots in the respective SAR subsections.

We are aware that this small set of examples may not be representative for the entire range of different kinds of signatures in SAR imagery caused by various oceanic and atmospheric phenomena. However, our analysis shows that there seems to be a basic difference in the curves of $D(i)$ dependent on the main spatial characteristics of the observed feature. That is, large- and mesoscale phenomena, such as the atmospheric convective cells and the large sea surface area covered by natural slicks, seem to exhibit a broader curve of $D(i)$ without a second maximum, whereas small-scale, more "patchy" features, like oil spills and rain cells, tend to give rise to a second maximum, which may be more or less pronounced, depending on the shape of the particular feature.

The application of an adaptive speckle filter (like the Kuan filter; see the bright, pink curves in Fig. 4) leads to a reduction of the second maximum of the $D(i)$ curves, especially for the "patchy" features, like oil spills and rain cells (Panels a), b), and e), respectively). It is also noteworthy that the speckle filter causes a smaller difference in the $D(i)$ curves of the large- and meso-scale phenomena (convective cells and slicks, Panels $\mathrm{c}$ ) and d)). Finally, the differences in the maximum fractal dimension are better visible after having applied a speckle filter.

\section{SUMMARY AND CONCLUSIONS}

In the framework of the joint European project "Clean Seas", the oil pollution of the southern Baltic Sea, the North Sea, and the northwestern Mediterranean Sea has been studied within a two-year period from December 1996 until November 1998. Our statistical analyses clearly show that satellite-borne SAR is an appropriate
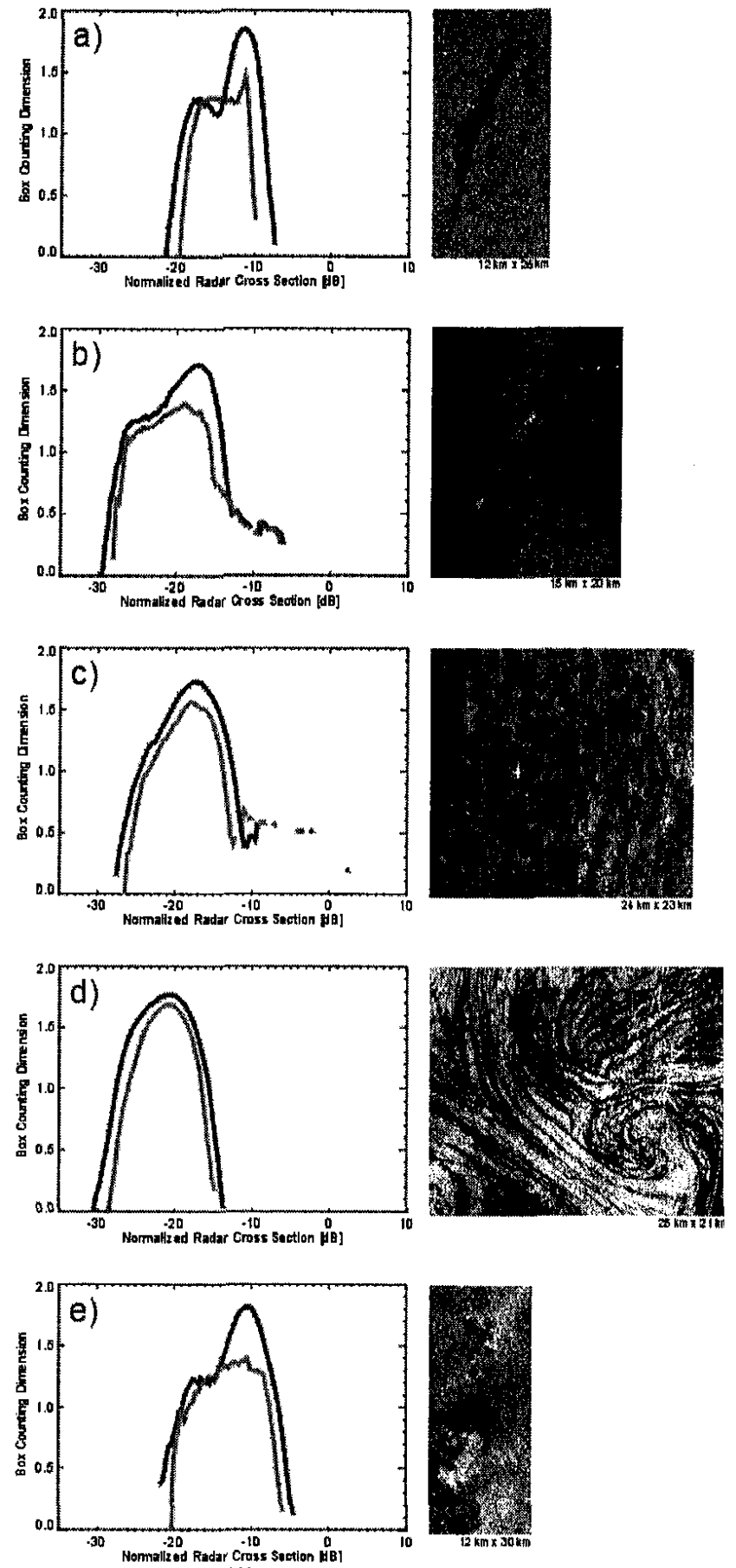

Fig. 4: Five different sections of ERS-2 SAR images corresponding to different oceanic and atmospheric phenomena. Also plotted are the respective functions $D(i)$ without (dark, purple) and after applying a speckle-noise (Kuan) filter (bright, pink): a,b) anthropogenic oil spills, c) atmospheric convective cells, d) biogenic sea slicks, and e) rain cells. 
system for routine oil spill detection, both in coastal areas and along ship routes. However, due to the poor coverage in time we are still far from using satellite-borne SAR imagery as a sufficient tool for oil spill monitoring.

We have introduced an advanced image processing technique for the classification of the observed radar signatures, namely the calculation of the fractal dimension. There seem to be differences in the maximum fractal (box counting) dimension of oil spills compared to other types of oceanic phenomena, probably due to the short span of the spills. We believe that by using fractal analysis techniques existing oil spill detection algorithms may be significantly improved. Finally, our results show imply that even the presence of a ship (or any other small bright feature) within a given SAR subsection can be inferred from the high-backscatter "tail" of the calculated $D(i)$ curves.

\section{ACKNOWLEDGMENTS}

The authors are grateful to J. Scholz for his help in generating the figures and to G.W. Jolly for the coordination of the Clean Seas project. This work has been supported by the European Community (EC) under contract ENV4-CT96-0334, "Clean Seas".

\section{REFERENCES}

[1] Gade, M., W. Alpers, H. Hühnerfuss, H. Masuko, and T. Kobayashi, The imaging of biogenic and anthropogenic surface films by the multi-frequency multipolarization SIR-C/X-SAR, J. Geophys. Res., 1885118866, 1998.

[2] Gade, M., and W. Alpers, "Using ERS-2 SAR images for routine observation of marine pollution in European coastal waters," Sci. Total Environ., in press, 1999.

[3] Redondo, J.M., "Fractal description of density interfaces," Institute of Mathematics and its Applications, vol. 56, 210-218, 1996. 